\title{
Physical Examination Numeric Result in Standard Unit
}

National Cancer Institute

\section{Source}

National Cancer Institute. Physical Examination Numeric Result in Standard Unit. NCI

Thesaurus. Code C83360.

The numerical identifier of a physical examination result in standard units. 